\title{
Nursing Time Devoted to Medication Administration in Long-Term Care: Clinical, Safety, and Resource Implications
}

\author{
Mary S. Thomson, PhD, ${ }^{*}$ Andrea Gruneir, PhD, ${ }^{*}$ Monica Lee, MSc, ${ }^{*}$ Joann Baril, BS, ${ }^{\dagger}$ \\ Terry S. Field, DSc, Jerry H. Gurwitz, MD, ${ }^{\dagger}$ and Paula A. Rochon, MD, MPH*
}

OBJECTIVES: To quantify the time required for nurses to complete the medication administration process in longterm care (LTC).

DESIGN: Time-motion methods were used to time all steps in the medication administration process.

SETTING: LTC units that differed according to case mix (physical support, behavioral care, dementia care, and continuing care) in a single facility in Ontario, Canada.

PARTICIPANTS: Regular and temporary nurses who agreed to be observed.

MEASUREMENTS: Seven predefined steps, interruptions, and total time required for the medication administration process were timed using a personal digital assistant.

RESULTS: One hundred forty-one medication rounds were observed. Total time estimates were standardized to 20 beds to facilitate comparisons. For a single medication administration process, the average total time was $62.0 \pm 4.9$ minutes per 20 residents on physical support units, $84.0 \pm 4.5$ minutes per 20 residents on behavioral care units, and $70.0 \pm 4.9$ minutes per 20 residents on dementia care units. Regular nurses took an average of $68.0 \pm 4.9$ minutes per 20 residents to complete the medication administration process, and temporary nurses took an average of $90.0 \pm 5.4$ minutes per 20 residents. On continuing care units, which are organized differently because of the greater severity of residents' needs, the medication administration process took $9.6 \pm 3.2$ minutes per resident. Interruptions occurred in $79 \%$ of observations and accounted for $11.5 \%$ of the medication administration process.

CONCLUSION: Time requirements for the medication administration process are substantial in LTC and are compounded when nurses are unfamiliar with residents. Interruptions are a major problem, potentially affecting the

From the * Kunin Lunenfeld Applied Research Unit, Baycrest Centre, Toronto, Ontario, Canada; and ${ }^{\dagger}$ Meyers Primary Care Institute, University of Massachusetts Medical School, Fallon Clinic, and Fallon Community Health Plan, Worcester, Massachusetts.

Address correspondence to Paula A. Rochon, Kunin Lunenfeld Applied Research Unit, Baycrest, 3560 Bathurst St., Toronto, Ontario, Canada, M6A

2E1. E-mail: paula.rochon@utoronto.ca

DOI: $10.1111 /$ j.1532-5415.2008.02101.x efficiency, quality, and safety of this process. J Am Geriatr Soc 57:266-272, 2009.

Key words: time-motion; medication administration; nursing time; long-term care; safety

$M$ edications are the most common therapeutic intervention used in long-term care (LTC). Although medication administration may seem to be a simple nursing task, the combination of polypharmacy and medical complexity in this population means that it is often more complex than fully realized..$^{1-3}$ The presence of cognitive, behavioral, or swallowing problems in the recipient may complicate even the act of directly administering a single medication. These problems are common in the LTC population. ${ }^{4,5}$

Within LTC, the low nurse-to-resident ratio often means that a single nurse is responsible for the care and safety of a large number of highly vulnerable and clinically complex patients. Performing treatments, filling out charts, and meeting with families and other care providers are just a few of the other demands on limited nursing time. ${ }^{6}$ Adequate time for medication administration is essential for improvements in medication safety, because this has been identified as the period when medication errors are most likely to occur. ${ }^{7,8}$ Furthermore, appropriate time allocations for medication administration may affect resident satisfaction and the quality of staff-resident interactions.

Despite being recognized as a fundamental nursing task and a key aspect of resident safety, ${ }^{6}$ little is known about the time requirements for medication administration. Additionally, no research has identified what steps in the medication administration process are the most time consuming and whether this differs according to resident characteristics. Understanding the time required for this task is an important component of any strategy to improve the efficiency and safety of medication administration.

The purpose of this study was to quantify the time required for the full medication administration process in in- 
stitutional LTC. Time-motion methods were used to record the amount of time nursing staff took to complete the medication administration process, from arranging the medication cart to completion of medication administration duties. To determine whether resident or nurse characteristics influenced time required to complete these tasks, medication rounds were observed in four unit types, and regular and temporary nurses were observed.

\section{METHODS}

\section{Study Design}

Time-motion methods were used to observe nurses directly during the medication administration process and to quantify the time required to complete each of seven steps in the process (defined below). In time-motion studies, an observer follows a subject and continually records the nature and duration of every activity with a timing data collection tool. ${ }^{9-12}$ As far as the authors are aware, this is the first study of its kind.

\section{Setting and Participants}

This study was conducted in a single, large, not-for-profit, academically affiliated LTC facility in Toronto, Ontario. The facility consists of 472 standard LTC beds and 300 specialized continuing care beds. This study was conducted on four unit types that differed according to intensity of nursing care required and specific resident characteristics. The unit types were defined as physical support (designed for residents with minimal cognitive or behavioral problems but who require medical monitoring and assistance with activities of daily living), behavioral and mental health care (designed for residents with a history of disruptive or aggressive behaviors), dementia care (designed for residents with moderate to severe dementia and who wander or exhibit occasional inappropriate behaviors), and continuing care (designed for residents who require extensive medical supervision and assistance with activities of daily living). Continuing care units have higher nurse-to-resident ratios than the other unit types.

\section{Nurse Types}

Registered nurses and registered practical nurses were observed during the study. Regular nurses were defined as those regularly assigned to the observed unit. Temporary nurses were defined as those not regularly assigned to the observed unit and for whom this was their first experience on the unit; this included nurses who regularly worked on other units but were temporarily reassigned to the unit on the day of observation and nurses who were part of a pool of float nurses who had no regular unit assignment. All residents who were scheduled to receive medication during the observed medication administration processes were included.

\section{Data Collection}

All observations were timed using a personal digital assistant (PalmOne, Inc., Milpitas, CA) with timer software (Stevens Creek Software, LLC, Cupertino, CA). The observer continuously timed each observation and tapped codes that corresponded to the nurse's actions. Interrater reliability was not assessed because a single observer conducted all observations.

\section{Medication Administration Time Periods}

One hundred twenty-six regularly scheduled medication administration time periods by regular nurses, evenly distributed across morning, noon, and evening time periods, were observed. Morning medication time periods took place between approximately 7:30 a.m. and 9:30 a.m., noon medication time periods between 12:00 p.m. and 12:30 p.m., and evening medication time periods between 4:00 p.m. and 5:30 p.m. An additional 15 regularly scheduled medication administration time periods delivered by temporary nurses were observed. These were also stratified according to time of day (morning $(\mathrm{n}=5)$, noon $(\mathrm{n}=6)$, and evening $(\mathrm{n}=4))$.

\section{Medication Administration Process Definition and Steps}

The research team developed a seven-step time-motion protocol based on discussion with facility staff and observations on units where the study was conducted. For the purpose of this study, the medication administration process was defined as the time that the nurse spent with the medication cart at regularly scheduled intervals. This definition did not encompass all medication-related activities (such as documentation in the medical record and discussions with pharmacy staff and other healthcare professionals on the floor or over the telephone) that occurred outside of those regularly scheduled intervals. Before study initiation, the time-motion protocol was pilot-tested on six medication administration processes. The protocol steps were refined based on these findings. The steps in the medication administration process were organize medication cart and obtain supplies, locate and identify resident, prepare medications (check medication administration record, dispense, and alter dosage form (usually by crushing)), prepare resident to receive medication, provide medication to resident, observe resident after receipt of medication to assess for any immediate adverse event, and travel back to medication cart. A more complete description of each step is provided in Appendix A.

Interruptions were recorded and were defined as any demand that caused the nurse to deviate from medication administration activities. These included telephone calls, questions from other staff, and resident emergencies.

One author (MT) conducted all of the observations. Before each observation, the observer made appointments with nurses and explained the purpose of the study. Each resident's medication administration record was obtained to provide a count of the total prescribed medications at each time of day. Nurses were instructed to perform the medication administration process as usual. The observer did not interact with nurses or residents.

\section{Analysis}

The average time required for the total medication administration process and for each predefined step in the process was calculated. To determine whether time varied according to specific factors, analyses were stratified according to unit type, time of administration (morning, noon, and evening), and nurse type (regular and temporary). Standard- 
ization was used to obtain a 20-bed unit estimate of the average time required for the total medication administration process. To do this, the observed average minutes per resident estimate was multiplied by 20 . Standardization to 20 beds was necessary to facilitate comparison between units and to improve generalizability of findings. A 20-bed standard was chosen, because this is the average unit size in LTC facilities. Because of differences in their organization and staffing policies, estimates for continuing care units were not standardized.

The percentage of time engaged in the medication administration process during a day shift was calculated by dividing the standardized average total recorded time for the morning and noon time periods by the total amount of time nurses spent on the units ( 7 hours when time for lunch and breaks are excluded). The day shift is from 7 a.m. to 3 p.m. and includes the regularly scheduled morning and noon medication administration process time periods. These calculations do not include time spent on medication activities outside of the predefined medication administration process such as pro re nata (PRN or as needed) medications or scheduled afternoon time periods. Again, these estimates do not include continuing care units.

Analyses were performed using SAS, version 9.1 (SAS Institute, Inc., Cary, NC).

\section{Ethics and Informed Consent}

Approval was obtained from the facility's research ethics board. Permission to conduct the study was obtained from the Vice President of Medical Services, the Vice President of Collaborative Practice, the Quality and Chief Nursing Executive, Unit Directors, and nursing union representatives. Each observed nurse signed a consent form after receiving a full explanation of the study purpose and methods.

\section{RESULTS}

In total, 141 medication administration processes were observed over an 11-month period. The full medication administration process, from organizing the medication cart to completion, was lengthy but varied according to unit type and time of day (Table 1).

Table 1. Long-Term Care (LTC) Unit Type Descriptors Including Mean Observed Total Time (Minutes) and per Resident Time for the Complete Medication Administration Process

\begin{tabular}{|c|c|c|c|c|}
\hline \multirow{2}{*}{$\begin{array}{c}\text { Medication Administration } \\
\text { Time Period }\end{array}$} & \multicolumn{4}{|c|}{ Unit Type } \\
\hline & Physical Support & Behavioral Care & Dementia Care & Continuing Care \\
\hline Number of residents, mean \pm SD & $21.2 \pm 4.6$ & $18.2 \pm 4.1$ & $22.5 \pm 5.1$ & $6.5 \pm 0.6$ \\
\hline $\begin{array}{l}\text { Number of medications administered, } \\
\text { mean } \pm \mathrm{SD}\end{array}$ & $115.3 \pm 70.00$ & $81.4 \pm 51.4$ & $109.6 \pm 75.8$ & $39.3 \pm 15.9$ \\
\hline Minutes per resident, mean \pm SD & $3.1 \pm 1.1$ & $4.2 \pm 1.0$ & $3.5 \pm 1.1$ & $9.6 \pm 3.2$ \\
\hline Minutes per 20 beds, mean $\pm \mathrm{SD}^{*}$ & $62.0 \pm 4.9$ & $84.0 \pm 4.5$ & $70.0 \pm 4.9$ & $N A^{\dagger}$ \\
\hline Seconds per medication, mean \pm SD & $40.7 \pm 20.3$ & $67.8 \pm 19.7$ & $55.6 \pm 19.2$ & $103.6 \pm 30.5$ \\
\hline \multicolumn{5}{|l|}{ Morning } \\
\hline Minutes per resident, mean \pm SD & $3.9 \pm 0.6$ & $5.2 \pm 0.7$ & $4.3 \pm 1.1$ & $12.9 \pm 2.6$ \\
\hline Minutes per 20 beds, mean $\pm \mathrm{SD}^{*}$ & $78.0 \pm 2.7$ & $104.0 \pm 3.1$ & $86.0 \pm 4.9$ & $N A^{\dagger}$ \\
\hline \multicolumn{5}{|l|}{ Noon } \\
\hline $\begin{array}{l}\text { Number of medications administered, } \\
\text { mean } \pm \text { SD }\end{array}$ & $52.3 \pm 9.6$ & $32.0 \pm 3.4$ & $35.7 \pm 7.6$ & $20.9 \pm 5.5$ \\
\hline $\begin{array}{l}\text { Observed total time, minutes, } \\
\text { mean } \pm S D(I Q R)\end{array}$ & $37.4 \pm 16.7$ (20.5-43.3) & $44.2 \pm 10.3(36.8-54.2)$ & $41.0 \pm 11.8(29.3-51.4)$ & $44.6 \pm 11.9(37.1-49.7)$ \\
\hline Minutes per resident, mean \pm SD & $2.3 \pm 0.9$ & $3.4 \pm 0.8$ & $2.5 \pm 0.6$ & $6.7 \pm 1.3$ \\
\hline Minutes per 20 beds, mean $\pm \mathrm{SD}^{*}$ & $46.0 \pm 4.0$ & $68.0 \pm 3.6$ & $50.0 \pm 2.7$ & $\mathrm{NA}^{\dagger}$ \\
\hline \multicolumn{5}{|l|}{ Evening } \\
\hline $\begin{array}{l}\text { Number of medications administered, } \\
\text { mean } \pm \text { SD }\end{array}$ & $89.2 \pm 9.3$ & $63.3 \pm 7.0$ & $84.3 \pm 14.0$ & $36.8 \pm 9.4$ \\
\hline
\end{tabular}

* Standardized estimates are based on minutes per resident for the total medication administration process.

${ }^{\dagger}$ Standardized estimates are not relevant to continuing care units because of differences in unit organization and staffing and therefore are not presented here.

$\mathrm{SD}=$ standard deviation; IQR $=$ interquartile range. 


\section{Unit Descriptions}

On the physical support, behavioral care, and dementia care units, nurses were responsible for providing medications to approximately 20 residents, but the average number of medications administered \pm standard deviation ranged from $81.4 \pm 51.4$ on behavioral care units to $115.3 \pm 70.0$ on physical support units. On a per resident basis, time for the full medication administration process was $3.1 \pm 1.1$ minutes on physical support units, $4.2 \pm 1.0$ minutes on behavioral care units, and $3.5 \pm 1.1$ minutes on dementia care units.

Continuing care units are designed for residents with the heaviest physical care needs and are staffed differently than the other LTC units. On these units, nurses were responsible for providing medications to an average of six residents and administered an average of $39.3 \pm 15.9$ medications. For the total medication administration process, the observed time was $9.6 \pm 3.2$ minutes per resident.

\section{Differences Between Medication Administration Time Periods}

On all units, the lengthiest medication administration process was recorded in the morning, when residents received the greatest number of medications (physical support, $214.4 \pm 15.4$ medications; behavioral care, $145.1 \pm 16.5$ medications; dementia care, $208.9 \pm 21.4$; medications; continuing care, $60.2 \pm 8.9$ medications). Residents received the fewest medications during the noon time period (physical support, $52.3 \pm 9.6$ medications; behavioral care, $32.0 \pm 3.4$ medications; dementia care, $35.7 \pm 7.6$ medications; continuing care, $20.9 \pm 5.5$ medications).

\section{Results Standardized to a 20-Bed Unit}

Once standardized to 20 beds, differences across units become more apparent (Table 1). The estimated mean total time per 20 residents was $62.0 \pm 4.9$ minutes for physical support units, $84.0 \pm 4.5$ minutes for behavioral care, and $70.0 \pm 4.9$ minutes for dementia care.

Consistent with the number of medications administered, the lengthiest medication administration process was the morning time period (physical support, $78.0 \pm 2.7 \mathrm{~min}$ utes, behavioral care, $104.0 \pm 3.1$ minutes, and dementia care, $86.0 \pm 4.9$ minutes), and the shortest was the noon time period (physical support, $46.0 \pm 4.0$ minutes, behavioral care, $68.0 \pm 3.6$ minutes, and dementia care, $50.0 \pm 2.7$ minutes).

Based on standardized estimates, the proportion of a 7 hour day shift spent on medication administration was estimated as $29.5 \%$ (interquartile range (IQR) $23.3-33.2 \%$ on physical support units, $40.9 \%$ (IQR $35.4-47.1 \%$ ) on behavioral care units, and $32.3 \%$ (IQR 26.3-34.7\%) on dementia care units.

Given differences in unit organization and staffing, standardized estimates are not relevant for continuing care units and are not presented here.

\section{Steps in the Medication Administration Process}

Figure 1 displays the length of time required for each step in the medication administration process and interruptions in seconds per resident. For each step, the most time was required on continuing care units, followed by behavioral care units, dementia care units, and physical support units. On all units, preparing medications and providing medications were the most time-consuming steps. These were also

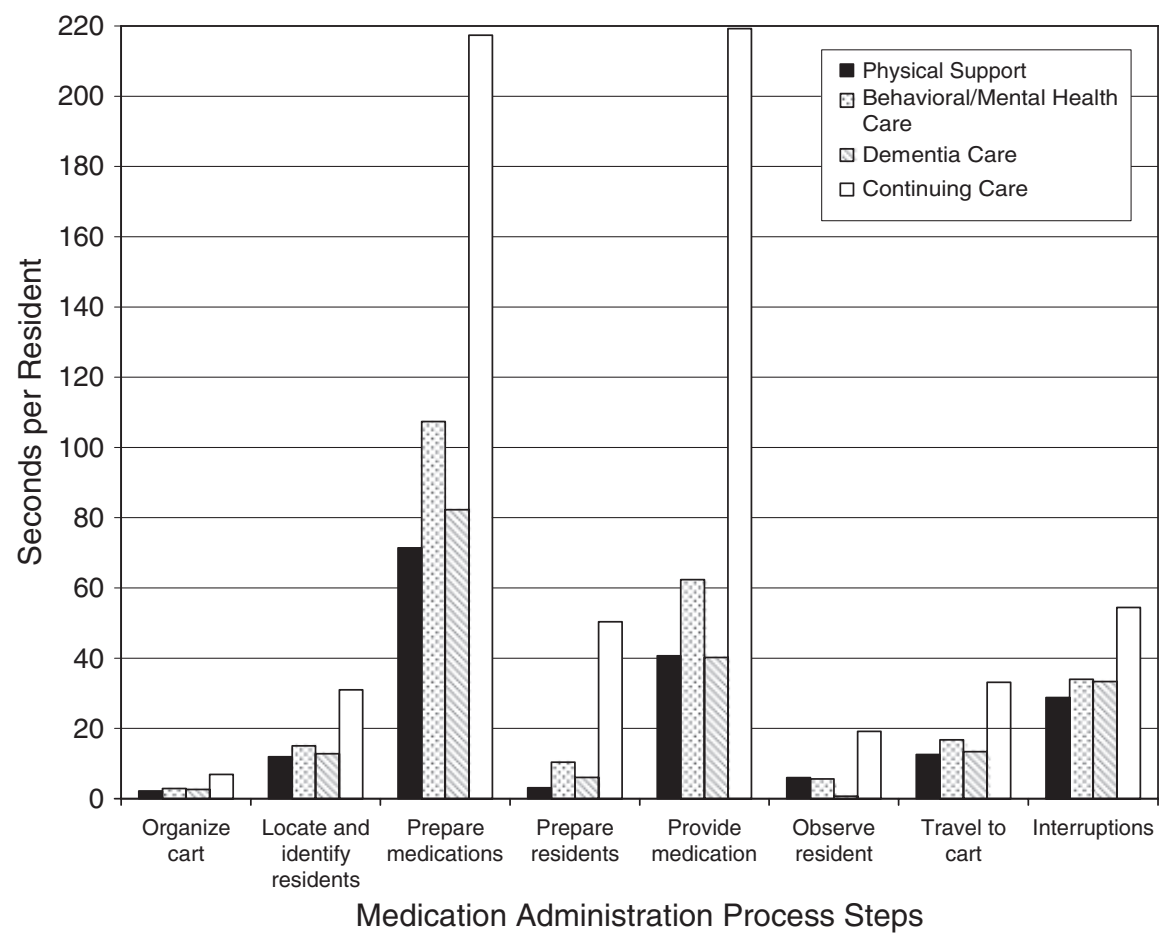

Figure 1. Average time recorded for each step in the medication administration process and interruptions stratified according to unit type for regular nurses only. Average time includes observations during morning, noon, and evening medication rounds on each unit type. Results presented as seconds per resident. 
the only steps directly affected by the number of medications to be administered.

\section{Interruptions}

Interruptions accounted for $11.5 \%$ of the total observed time. At least one interruption was recorded in $79 \%$ of medication rounds, but the distribution was skewed. An average of $4.8 \pm 6.6$ interruptions (median 3.0) were counted per medication administration process. On average, interruptions accounted for $8.2 \pm 11.2$ minutes, but this varied according to unit type (physical support, $13.8 \pm 18.3$ minutes; behavioral care, $10.6 \pm 10.7$ minutes; dementia care, $11.4 \pm 8.8$ minutes; continuing care, $3.7 \pm 5.7$ minutes).

\section{Temporary Versus Regular Nurses}

Temporary nurses took longer to complete the medication administration process than did regular nurses (temporary, $90.0 \pm 5.4$ minutes per 20 residents; regular, $68.0 \pm 4.9$ minutes per 20 residents). Temporary nurses took longer to complete each step in the medication administration process, especially identifying residents (temporary, $22.4 \pm 9.0$ seconds per resident; regular, $13.4 \pm 4.8$ seconds per resident) and preparing medications (temporary, 113.2 \pm 39.2 seconds per resident; regular, $87.5 \pm 36.2$ seconds per resident). Temporary nurses also took longer to address interruptions (temporary, $50.7 \pm 42.6$ seconds per resident; regular, $32.2 \pm 30.0$ seconds per resident) (Figure 2).

\section{DISCUSSION}

This study demonstrates that the medication administration process in LTC is time consuming. Within a 7-hour day shift on a 20-bed unit, which includes two regularly scheduled medication administration time periods, the medication administration process accounted for approximately onethird of nursing time. Although this is only slightly greater than the time hospital-based nurses were found to spend on medication administration, ${ }^{13}$ differences in staffing between the two settings make this critical. On a typical LTC unit with approximately 20 residents, there is only one nurse per shift. The nurse is responsible for overseeing the nursing aides as well as other aspects of care such as providing treatments, documenting in resident charts, and meeting with families and other staff. With so much time required for medication administration, the nurse is often less available to handle other tasks. Furthermore, research shows that residents receive only 15 to 20 minutes of direct care time from nurses, ${ }^{14}$ and the findings of the current study suggest that a large proportion of that time is spent on administering medication. This raises significant concerns about how the overall quality of care is affected when so much care time is devoted to a single task.

These estimates of the true amount of time that nurses in LTC spend on medication administration are conservative. The findings in this study did not include all regular medication administration time periods (such as midafternoon and bedtime) or time spent on medication activities outside of the regular medication administration times (such as PRN and off-schedule medications). Furthermore, these estimates do not include time spent on medicationrelated activities that were not included in the definition of the medication administration process. Such medicationrelated activities include discussing residents' medications with the physician, pharmacy, and family and documenting changes and problems with medications.

A substantial amount of time during the medication administration process was spent addressing interruptions. At least one interruption was recorded in nearly $80 \%$ of all observations and, on average, accounted for 10 minutes of

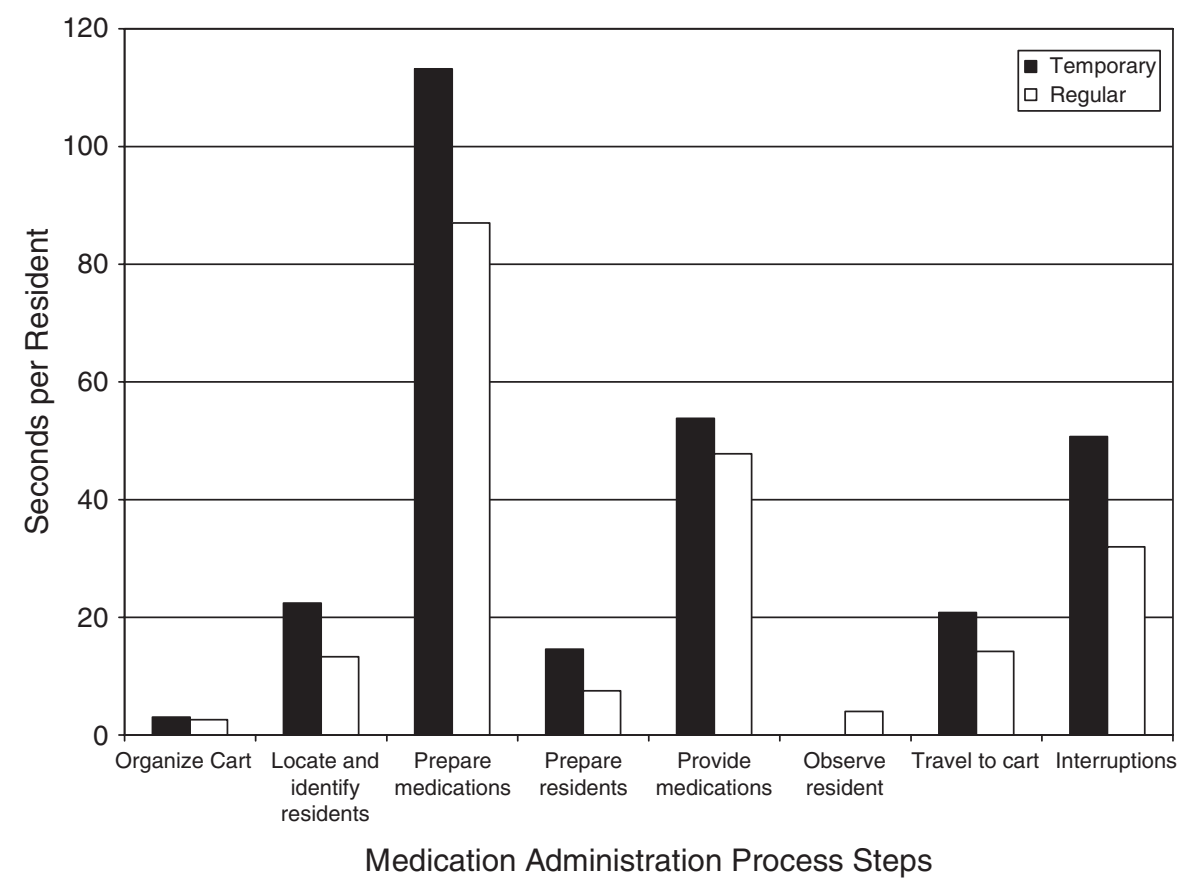

Figure 2. Average time recorded for each step in the medication administration process and interruptions for regular and temporary nurses. Average time includes observations during morning, noon, and evening medication rounds on each unit type. Results presented as seconds per resident. 
the medication administration process. Interruptions are not unique to LTC or the medication administration process. Studies in multiple healthcare settings, including operating rooms, ${ }^{15}$ intensive care units, ${ }^{16}$ and emergency departments, ${ }^{17}$ have found interruptions to be frequent and problematic. Research on the cognitive workflow of nurses has shown that interruptions were frequently associated with shifts in attention. ${ }^{18}$ Others have shown correlations between interruptions during medication administration and medication errors. ${ }^{19,20}$ Nurses have also reported concerns about the direct effect of interruptions on quality and on patients' perceptions of nursing care. ${ }^{21}$ In acute care settings, simple strategies, such as having nurses wear "Do Not Disturb" badges, have been shown to be successful in reducing the occurrence of interruptions during medication administration, ${ }^{22-24}$ but even such simple strategies require that other nurses be available to address emergencies. This may at least partially explain why fewer interruptions were recorded on the continuing care units, where there is a higher nurse-to-resident ratio and more staff available to manage emergencies.

Temporary nurses took an average of 20 minutes more than regular nurses to complete the medication administration process and took longer to complete each step and resolve interruptions. It is likely that these findings are an underestimate of the extra time required by temporary staff, because the temporary nurses observed in this study were facility staff and had some familiarity with the units, unlike other facilities where temporary nurses are contracted from outside agencies. The use of temporary nurses, also known as agency or contract nurses, is increasingly common in North American nursing homes. ${ }^{25}$ In light of this, these findings raise broader questions about the costs and benefits of this staffing practice. Research on the use of temporary nurses and quality of care is inconclusive but suggests a complex relationship that other staffing factors may modify. ${ }^{25-27}$ With respect to medication-related activities, one study found that temporary nurses were less likely than regular nurses to be involved in errors. Regular nurses may omit certain steps, such as double-checking resident identification, to meet time pressures ${ }^{28}$ but temporary nurses, who are not familiar with residents or their routines, may not have this option.

The observed patterns across unit types suggest that the time required for the medication administration process increased as the degree of cognitive, behavioral, and physical needs intensified. Although individual resident characteristics were not measured, the variation across unit types illustrates how resident case mix can affect the time required for nurses to complete activities such as the medication administration process.

Medication administration has been identified as the most vulnerable part of the entire medication use process, which includes the full prescription-dispensation-administration cascade. ${ }^{7,8,28}$ Efforts to improve medication safety should address areas of vulnerability in the process, most notably the issue of interruptions. ${ }^{15}$ Strategies such as making the medication administration process a dedicated task may help to reduce interruptions and their potential effect. Adequate time will help to ensure that nurses are able to abide by medication safety protocols. In addition, strategies to support nurses during medication-related activities, such as making another nurse available during this time, may free up nurse time for other patient-care activities.

\section{Limitations}

It is possible that the presence of the observer influenced nurse behavior. Although it is not known whether or how this affected time measurements, it is unlikely that this was a serious problem, because nurses were explicitly informed that their performance was not being evaluated and that only time for completion was of interest. In addition, nurses had no control over the occurrence of interruptions. The measurements included only specific regularly scheduled medication administration time periods and did not include other regularly scheduled time periods, off-schedule medications, or other medication-related activities such as documentation. Therefore, the estimates presented here are an underestimate of the full time that nurses in LTC spend on medication activities. Similarly, the time-motion methods allowed for coding of only one activity at a time, and multitasking was not captured (e.g., if a nurse was speaking to someone while performing one of the steps in the medication administration process).

Finally, a single observer conducted this study in a single, large LTC facility. Despite this, it is likely that these findings are relevant to other LTC settings for three main reasons. First, a specifically defined medication administration process was focused on that should be similar across facilities. Second, data were presented according to unit type rather than overall so that readers can identify relevant groups. Finally, this study has illustrated that medication administration is a time-consuming process and that interruptions are a frequent occurrence; it is unlikely that these findings are limited to the study facility. Future studies are needed to better describe the effect of these issues on medication administration errors.

\section{CONCLUSION}

In LTC, the medication administration process accounts for a substantial portion of nursing time. It is likely that the findings from this study are an underestimate, because only the time when nurses were directly involved in administering medications was observed. Time for this activity is longer for temporary nurses, who may have less familiarity with residents and their routines. Interruptions are a major concern that may affect efficiency and safety. The medication administration process should be made a dedicated activity, and nurses should receive additional support to manage interruptions during these times.

\section{ACKNOWLEDGMENTS}

The authors would like to acknowledge Jackie Fuller for her help in conducting the statistical analyses and Peter Anderson for his assistance in coordinating the study. The authors would also like to thank all the nurses who kindly agreed to be observed and all the nursing staff who gave permission to conduct the study. We thank Joy Richards, PhD, Vice President Collaborative Practice, Quality, and Chief Nursing Executive for providing her comments on the study design and manuscript.

Conflict of Interest: The editor in chief has reviewed the conflict of interest checklist provided by the authors and has 
determined that the authors have no financial or any other kind of personal conflicts with this manuscript. This study was supported by grants from the Agency for Healthcare Research and Quality (HS010481 and HS15430). The contents are solely the responsibility of the authors and do not necessarily represent the official views of the Agency for Healthcare Research and Quality.

Author Contributions: Mary Thomson: acquisition of subjects and data, analysis, and interpretation of data and preparation of manuscript. Andrea Gruneir: analysis and interpretation of data and preparation of manuscript. Monica Lee: study concept and design and preparation of manuscript. Joann Baril: data analysis and preparation of manuscript. Terry Field and Jerry Gurwitz: study concept and design, analysis and interpretation of data and preparation of manuscript. Paula Rochon: study concept and design, acquisition of subjects and data, analysis and interpretation of data and preparation of manuscript.

Sponsor's Role: The sponsors played no role in the design, methods, data collection, analysis, or interpretation of this manuscript.

\section{REFERENCES}

1. Eisenhauer LA, Hurley AC, Dolan N. Nurses' reported thinking during medication administration. J Nurs Scholarship 2007;39:82-87.

2. Holmes HM, Hayley DC, Alexander CG. Reconsidering medication appropriateness for patients late in life. Arch Intern Med 2006;166:605-609

3. Gurwitz JH. Polypharmacy: A new paradigm for quality drug therapy in the elderly? Arch Intern Med 2004;164:1957-1959.

4. Griffith R. Managing difficulties in swallowing solid medication: The need for caution. Nurse Prescrib 2005;3:201-203.

5. Wright D. Medication administration in nursing homes. Nurse Standard 2002;16:33-38.

6. Bowers BJ, Lauring C, Jacobson N. How nurses manage time and work in long-term care. J Advanced Nurs 2001;33:484-491.

7. Pierson S, Hansen R, Greene S et al. Preventing medication errors in long-term care: Results and evaluation of a large scale web-based error reporting system. Qual Safety Health Care 2007;16:297-302.

8. U.S. Pharmacopeia, USP Patient Safety CAPSLink. Distractions Contribute to Medication Errors. 2003 [on-line]. Available at http://www.usp.org/pdf/EN/ patientsafety/capslink2003-09-01.pdf Accessed November 7, 2007.

9. Lo HG, Newmark LP, Yoon C. Electronic health records in speciality care: A time-motion study. J Am Med Informat Assoc 2007;14:609-615.

10. Finkler SA, Knickman JR, Hendrickson G et al. A comparison of work-sampling and time-and-motion techniques for studies in health services research. Health Serv Res 1993;28:577-597.

11. Barker KN. Data collection techniques: Observation. Am J Hosp Pharm 1980;37:1235-1243.

12. Burke TA, McKee JR, Wilson HC et al. A comparison of time-and-motion and self-reporting methods of work measurement. J Nurs Admin 2000;30:118-125.

13. Keohane CA, Bane AD, Featherstone E et al. Quantifying nursing workflow in medication administration. J Nurs Admin 2008;38:19-26.

14. Arling G, Kane R, Mueller $\mathrm{C}$ et al. Nursing effort and quality of care for nursing home residents. Gerontologist 2007;47:672-682.

15. Wiegmann DA, Elbardissi AW, Dearani JA et al. Disruptions in surgical flow and their relationship to surgical errors: An exploratory investigation. Surgery 2007;142:658-665.

16. Tang Z, Weavind L, Mazabob J et al. Workflow in intensive care unit remote monitoring: A time-and-motion study. Crit Care Med 2007;35:20572063.

17. Brixey JJ, Robinson DJ, Tang $Z$ et al. Interruptions in workflow for RNs in a level one trauma center. AMIA Annu Symp Proc 2005, 86-90.

18. Potter P, Wolf L, Boxerman S et al. Understanding the cognitive work of nursing in the acute care environment. J Nurs Admin 2005;35:327-335.

19. Scott-Cawiezell J, Pepper GA, Madsen RW et al. Nursing home error and level of staff credentials. Clin Nurs Res 2007;16:72-78.

20. Flynn EA, Barker KN, Gibson JT et al. Impact of interruptions and distractions on dispensing errors in an ambulatory care pharmacy. Am J Health-Syst Pharm 1999;56:1319-1325.
21. Beaudoin L, Edgar L. Hassles: Their importance to nurses' quality of work life. Nurs Econ 2003;21:106-113.

22. Bennet J, Harper-Femson LA, Tone J et al. Improving medication administration systems: An evluation study. Can Nurs 2006;102:35-39.

23. Pape TM. Applying airline safety practices to medication administration. Medsurg Nurs 2003;12:77-94.

24. Pape TM, Guerra DM, Muzquiz et al. Innovative approaches to reducing nurses' distractions during medication administration. J Contin Educ Nurs 2005;36:108-117.

25. Bourbonniere M, Feng Z, Angelelli J et al. The use of contract licensed nursing staff in U.S. nursing homes. Med Care Res Rev 2006;63:88-109.

26. Weech-Maldonado R, Meret-Hanke L, Neff MC et al. Nurse staffing patterns and quality of care in nursing homes. Health Care Manage Rev 2004;29:107115 .

27. Castle NG, Engberg J. The influence of staffing characteristics on quality of care in nursing homes. Health Serv Res 2007;42:1822-1847.

28. Vogelsmeier A, Scott-Cawiezell J, Zellmer D. Barriers to safe medication administration in the nursing home. Exploring staff perceptions and concerns about the medication use process. J Gerontol Nurs 2007;33:5-12.

\section{APPENDIX A}

Table A1. Nurse Activity During Each of the Predefined Steps in the Medication Administration Process

1 Organize medication cart Nurse begins preparations for the complete medication administration process; activities include checking current medication administration record and changes in physician orders; checking that medication type, dose, and time of administration are correct; organizing cart according to resident if not already done; gathering antibiotics, nonprescription medications, narcotics, and other specialty items from storage location; and gathering other supplied as needed (e.g., applesauce, jam).

2 Locate and identify Nurse identifies individual resident and resident checks resident room number and armband or identification against medication administration record.

3 Prepare medications Nurse prepares the medications to be provided to the identified resident: doublechecking the medication administration record, assembling medications according to instructions, altering medication dosage form if required (i.e., crushing or splitting pills), and obtaining water or other needed supplies.

4 Prepare resident to receive medications

Nurse conducts a basic assessment of the resident, which may include checking the resident's pulse, temperature, or other vital signs.

5 Provide medications to Nurse gives each medication in the the resident appropriate dosage form to the resident and signs the medication administration record.

6 Observe resident after providing medication

Nurse observes the resident after receiving medications to assess for any immediate adverse effects.

7 Travel back to medication cart

Nurse travels back to the medication cart after completing each step in the identified resident's medication administration process.

8 Interruptions Any unplanned demand that caused the nurse to deviate from the steps in the medication administration process, including phone calls from other departments, questions from other staff or residents, and emergencies. 